Journal of Computer Science 3 (8): 600-607, 2007

ISSN 1549-3636

(C) 2007 Science Publications

\title{
Automated Breast Cancer Diagnosis based on GVF-Snake Segmentation, Wavelet Features Extraction and Neural Network Classification
}

\author{
Abderrahim Sebri, Jihène Malek, Rached Tourki \\ Laboratoire d'Electronique et de Microélectronique, Département de Sciences Physiques, \\ Faculté des Sciences, 5019 Monastir, Tunisia
}

\begin{abstract}
Breast cancer accounts for the second most cancer diagnoses among women and the second most cancer deaths in the world. In fact, more than 11000 women die each year, all over the world, because this disease. The automatic breast cancer diagnosis is a very important purpose of medical informatics researches. Some researches has been oriented to make automatic the diagnosis at the step of mammographic diagnosis, some others treated the problem at the step of cytological diagnosis. In this work, we describes the current state of the ongoing the $\mathrm{BC}$ automated diagnosis research program. It is a software system that provides expert diagnosis of breast cancer based on three step of cytological image analysis. The first step is based on segmentation using an active contour for cell tracking and isolating of the nucleus in the studied image. Then from this nucleus, have been extracted some textural features using the wavelet transforms to characterize image using its texture, so that malign texture can be differentiated from benign on the assumption that tumoral texture is different from the texture of other kinds of tissues. Finally, the obtained features will be introduced as the input vector of a Multi-Layer Perceptron (MLP), to classify the images into malign and benign ones.
\end{abstract}

Key words: Breast Cancer, GVF-Snake, Segmentation, Wavelet feature extraction, Neural Network, MLP classification.

\section{INTRODUCTION}

Medical automatic diagnosis is still considered as a hard task. In fact, medical diagnosis requires an expert able to cope with the uncertain cases only by eyeing the visible symptoms. Such performances are difficult to achieve using an automatic system for diagnosis. Breast cancer is a vital problem needing quick handling and treatments. The cytological step of the diagnostic is the first step done to make a first approach of the case. It consists of the extraction of some cells of the lesion. Various histological tests are performed to make a first appreciation of the case but the ultimate diagnosis may be sometimes difficult to obtain, even for a medical expert.

This paper summarizes the current state of the Autonomous Diagnostic of Breast cancer Chip project, an ongoing interdisciplinary research effort begun at the University of Sciences of Monastir-Tunisia in the early 2000 's ${ }^{[1,2]}$. The project addresses an important problem in breast cancer treatment, that the diagnosis (determination of benign from malignant cases). This project is divided in two big parts of active research; the first is realization of solution software that permits the validation of the concept of the diagnosis of the breast cancer and the second is of built-in this process within a Hardware System "BioChips" for the detection [3], extraction and classification typical of the cancerous cells of a breast cancer from a cytological image. The resulting software system provides accurate and interpretable results to both doctor and patient to aid in the various decision-making steps in the diagnosis and treatment of the disease.

Many studies have been oriented to do pattern breast cancer diagnosis processes such as Xcyt Project $[4,5,6]$ is a software system that provides expert diagnosis and prognosis of breast cancer based on fine needle aspirates. The system combines techniques of digital image analysis, inductive machine learning, mathematical programming, and statistics, including novel prediction methods developed specifically to make best use of the cytological data available. The point is that the digitizing of the observed samples of lesions and their preprocessing leads to images on which the cells have different shapes and sizes, they are sometimes condensed and the edges and limits of each one can't often be detected. There are also residual cells which hadn't any thing to do with the lesion such as

Corresponding Author: Abderrahim Sebri, Laboratoire Electronique et Microélectronique Faculté des Sciences de Monastir 5019 Monastir, Tunisia 
blood ones for example (Figure 2). This entire make the process of classification of the images into benign or malign cases very difficult because of the absence of an easy mathematical formalism that can characterize the objects in the observed images.

Considering these constraints, the adopted software solution in this work is performed in three steps: a first, an active contour cell detecting technique is used to approximately isolate nucleus in an observed image. Then a texture-analysis-based feature extraction is done to the obtained nuclei. In fact, the wavelet transforms has been widely used for description of images texture $^{[7,8]}$. Numerous decompositions schemes have been proposed for the purpose of texture characterization ${ }^{[9]}$, two approaches can be detected: the first is numerical description mainly based on second order or higher statistics of the wavelet coefficients. The second approach is quantification of the textures in the way human can see them as coarse, regular, directional, symmetric, etc.

In this paper, the work is organized as follows (Figure 1); the first part is given the description of segmentation results of the technique of cancerous cell detected to basis of the Active Contour Models "Snake" ${ }^{[10]}$ and "GVF-Snake" ${ }^{[11,12]}$. The second part has led to nineteen textural features based discreet wavelet transform. Finally, the obtained features are used to achieve the classification of Images using their texture descriptions; in texture classification the goal is to assign an unknown sample image or an image block to one of a set of known texture classes. This last work is assured by the application of the Neural Network classification as MLP (Multi-Layer Perceptron).

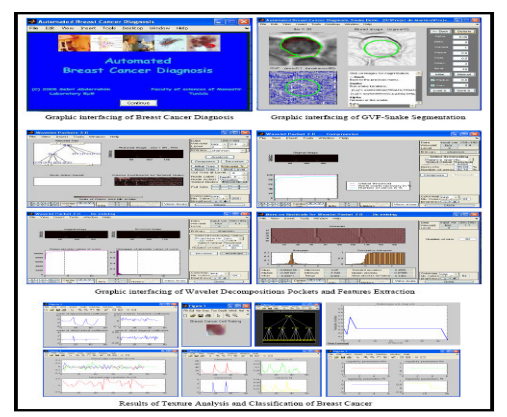

Graphic interfacing of Breast Cancer Diagnosis

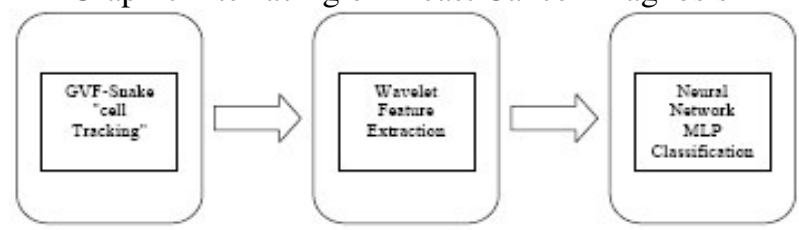

Fig. 1: The followed steps diagnostic of breast cancer to image classification into malign and benign images

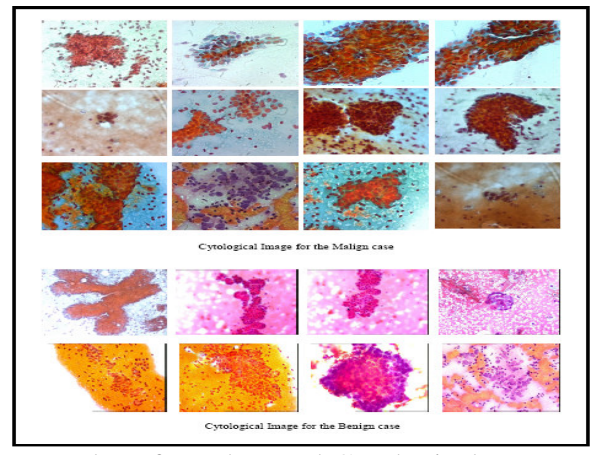

Fig. 2: Examples of an observed Cytological Image of Breast Cancer samples: (a) Cytological Image for the malign case, (b) Cytological Image for the benign case

Background on Active Contour Models or "Snake"and "GVF-Snake": Kass and al. ${ }^{[10]}$ proposed active Contour Models, also called snakes, that since then have been successfully applied in a variety of problems in computer vision and image analysis, such as edge and subjective contours detection, motion tracking and segmentation ${ }^{[13]}$. Basically, there are two types of snake models: the implicit ones and the parametric ones.

The parametric snake models consist basically of an elastic curve (or surface), which can dynamically conform to object shapes in response to internal forces (elastic forces) and external forces (image and constraint forces). These forces can be the result of a functional global minimization processor based on local information. Such approach is more intuitive than the implicit models. Its mathematical formulation makes easier to integrate image data, an initial estimated, desired contour properties and knowledge-based constraints, in a single extraction process ${ }^{[13]}$.

However, parametric models have also their limitations. First, most of these methods can only handle topologically simple objects. The topology of the structures of interest must be known in advance since the mathematical model cannot deal with topological changes without adding extra machinery ${ }^{[14,15]}$. Second, parametric snakes are too sensible to their initial conditions due to the non-convexity of the energy functional and the contraction force, which arises from the internal energy term ${ }^{[17,18]}$.

Several works have been done to address these limitations. The use of simulated annealing for minimization and dynamic programming ${ }^{[19]}$ has been proposed to reduce problems caused by convergence to local minima. However, the utility of such techniques is limited by performance problems ${ }^{[17,20]}$. Levine. al. ${ }^{[21]}$ used another approach by applying hierarchical filtering methods, as well as a continuation method based on a 
discrete scale-space representation. Basically, a scalespace scheme is first used at a coarse scale to get closer to the global energy minimum represented by the desired contour. In further steps, the optimal valley or contour is sought at increasingly finer scales. These methods address the no convexity problem but not the bad effects of the internal normal force. This force is a contraction force, which makes the curve collapse into a point if the external field is not strong enough.

In Cohen.al ${ }^{[22]}$ and Gang Xu. al. ${ }^{[18]}$ This problem is addressed by the addition of another internal force term to reduce the bad effects of the contraction force. In both these works the number of parameters are increased and there are some trade-offs between efficiency and performance. Another way to remove the undesired contraction force of the snake model is to use the idea of invariance, which is well known, in the field of computer vision ${ }^{[23]}$. That idea has been applied for closed contours and consists in designing an internal smoothing energy, biased toward some prior shape, which has the property of being invariant to scale, rotation and translation. In these models, the snake has no preference to expand or contract but tends to acquire a natural shape.

Gradient Vector Flow "GVF-Snake"(Parametric GVF-Snake Model): The convergence of Snakes contours depends on the interaction of internal and external forces, where the minimum energy can be found ${ }^{[10]}$. The internal force is based on the assumption that the contour is continuous and smooth, while the external force is used to extract the desired characteristics, for example a line or an object boundary. In other hand, the conventional classical Snakes model has some limitation in that the initial contour must be placed close to the object to prevent it from converging to a local minimum. To solve these problems the method of GVF-Snake is currently also most efficient to the point of view precision in the tracking breast cancer cells.

C. Xu and J.L. Prince ${ }^{[11,12]}$ proposed to generate a more general field by allowing the possibility that it comprises both an irrotational component and a solenoidal component. They designed a new force field that has both the desired properties of both a large capture range and the presence of forces that point into boundary concavities.

The gradient vector flow field $F_{\text {ext }}=V(x, y)$ is derived from the following energy functional in ${ }^{[11,12]}$ :

$E_{G V F}=\iint\left(u\left(u_{x}^{2}+u_{y}^{2}+v_{x}^{2}+v_{y}^{2}\right)+|\nabla f|^{2}|V-\nabla f|^{2}\right) d x d y$

Where $\mathrm{V}(\mathrm{x}, \mathrm{y})$ is the field vector Flow of image:

$V(x, y)=(v(x, y), u(x, y))$
In this context, $f$ is the edge map, and $\mu$ is a decreasing function of the gradient magnitude defined as ${ }^{[9,10]}$ :

$$
\begin{aligned}
& f(x, y)=\left|\nabla G_{\sigma}(x, y) * I(x, y)\right| \\
& \mu=\exp \left(-\left(\frac{|\nabla f|}{K}\right)\right)
\end{aligned}
$$

$K$ is a positive constant controlling the smoothness of the resulting field ${ }^{[11,12]}$. Calculus of variations is once again applied to Minimize (6) leading to the following Euler equations ${ }^{[14]}$ :

$$
\begin{aligned}
& \mu \nabla^{2} u(x, y)-\left(u(x, y)-f_{x}\right)\left(f_{x}^{2}+f_{y}^{2}\right)=0 \\
& \mu \nabla^{2} v(x, y)-\left(v(x, y)-f_{y}\right)\left(f_{x}^{2}+f_{y}^{2}\right)=0
\end{aligned}
$$

One solves (5) and (6) to obtain the GVF force field $(u, v)$ that minimizes (1). The resolution of these equations can be iterative to basis to transform it of Euler-Lagrange for the differential equations. Of which this method is adapted for the numeric treatment while considering $u_{t}$ and $v_{t}$ iterated data, with $t$ is the indication of iteration:

$u_{t}(x, y)=\mu \nabla^{2} u_{t}(x, y)-\left(u(x, y)-f_{x}\right)\left(f_{x}^{2}+f_{y}^{2}\right)$
$v_{t}(x, y)=\mu \nabla^{2} v_{t}(x, y)-\left(v(x, y)-f_{y}\right)\left(f_{x}^{2}+f_{y}^{2}\right)$

The deformable models are used extensively in image processing, computer vision, and medical imaging applications, particularly to delineate object boundaries. Problems associated with initialization and poor convergence to boundary concavities, however, has limited their utility. This section presents an external force for deformable models, largely solving both problems in cytological image segmentation. This external force, which we call gradient vector flow (GVF), is computed as a diffusion of the gradient vectors of a gray-level or binary edge map derived from the image. It differs fundamentally from traditional deformable model external forces in that it cannot be written as the negative gradient of a potential function, and the corresponding deformable model is formulated directly from a dynamic force equation rather than an energy minimization formulation (equation 1). Using several two-dimensional examples, we show that GVF has a large capture range and is able to move deformable models into boundary concavities.

Our objective has been performing a comparative study of traditional ${ }^{[10]}$ and gradient vector flow ${ }^{[11]}$ snake models for tracking cancers cells from cytological image of breast cancer ${ }^{[3]}$. First, we have evaluated the accuracy of the shape descriptions obtained by these snake models in gray-level images, showing that gradient vector flow snakes outperform traditional snake models. Next, we have evaluated the 
robustness of the two snake models with respect to changes of model parameter values, i.e., regularization, elasticity and weight of the external force parameters. Finally, we have evaluated the robustness of the snake models with respect to changes of user-defined parameters, such as the position, size and shape of the initial snake. Our experimental results have shown that, the gradient vector flow snake model outperforms other snake models in terms of robustness with respect to both model and user-defined parameters, followed closely by the classical snake model.

Particular advantages of the GVF deformable model over a traditional deformable model are its insensitivity to initialization and its ability to move into boundary concavities. As we show in Figure 5, its initializations can be inside, outside, or across the object's boundary (cancers cells). Unlike deformable models that use pressure forces, a GVF deformable model does not need prior knowledge about whether to shrink or expand toward the boundary. The GVF deformable model also has a large capture range, which means that, barring interference from other objects, it can be initialized far away from the boundary. This increased capture range is achieved through a spatially varying diffusion process which does not blur the edges themselves, so multi resolution methods are not needed.

Finally, in this section the isolation of the cells in breast cancer cytological image has been achieved, by the use of parametric active contour models. The obtained cells will be used to make the classification of the case into benign or malign breast lesion. In the next section, we introduce the step of Textural Feature Extraction based Discreet Wavelet Transform.

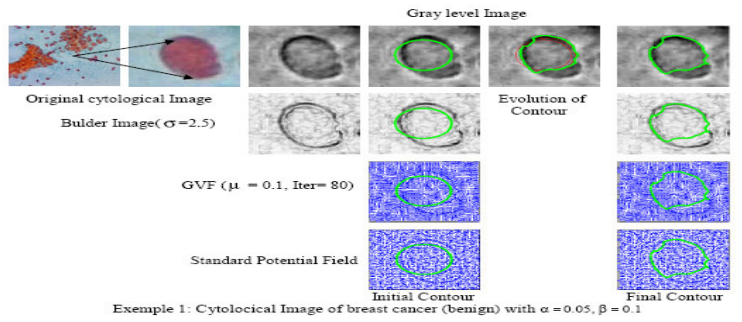

Fig. 3: Application of GVF-Snake Model for some types of Cytological Image of Breast Cancer

Analysis Texture Method based on Discreet Wavelet Transform: Introduction: Texture features provide a measure of the underlying texture within a given region. A variety of methods are used to extract texture characteristics. Some of the commonly used approaches include the use of spatial frequencies, edge frequencies, run lengths, pixel's joint probability distribution, and special masks such as Law's masks. It has been demonstrated in different studies that different feature extraction methods yield different results based on the application domain and it is for this reason that a diverse range of techniques were investigated. The evaluation of texture feature is an important step in the image processing of the studied images. The texture analysis is the basis of pattern recognition and classification especially in medical domain. The ability of classifiers depends on the quality of feature used as well as the amount of the data available to them ${ }^{[24]}$. There are many texture extraction methods such as autocorrelation based texture features, Co-occurrence matrices texture features ${ }^{[25]}$, edge-frequency based texture features, etc.

In this work, the texture analysis has been done using a based transform method of feature extraction. The used transforms in this paper, are discrete wavelet transforms. In all this work the obtained results have been achieved using two kinds of transforms: the first one is the Daubechies wavelet at level 2 (db2) which is an orthogonal and compactly supported wavelet, and the second one is the B-splines biorthogonal wavelet (bior) which is a biorthogonal and compactly supported pairs of wavelets. In the two cases the Fast wavelet transform (FWT) is used, with finite impulse filters (FIR filters), the first one is however an asymmetric wavelet with poor regularity and orthogonal proprieties while the biorthogonal wavelet is symmetric but without orthogonality.

For the $\mathrm{db} 2$ transform the high pass filter is $\mathrm{h}_{0}$ and the low pass filter is $\mathrm{h}_{1}$; given by:

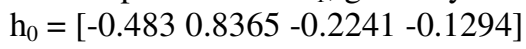

$\mathrm{h}_{1}=\left[\begin{array}{llll}-0.1294 & 0.2241 & 0.8365 & 0.483\end{array}\right]$

For the bior transform the high pass filter is $\mathrm{h}_{2}$ and the low pass filter is $\mathrm{h}_{3}$; given by:

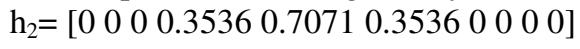

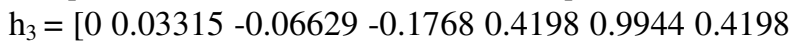

$-0.1768-0.06630 .0331]$

2D Discrete Wavelet Transforms (2D DWT Method): The classical 2D wavelet transform is performed by two separate 1D transforms along the rows and the columns of the image data, resulting at each step of decomposition in a low pass image or the coarse scale approximation and tree detail images as shown in the Figure 4.

\begin{tabular}{|c|c|}
\hline $\mathrm{LL}_{0}$ & $\mathrm{LH}_{0}$ \\
\hline $\mathrm{HL}_{0}$ & $\mathrm{HH}_{0}$ \\
\hline
\end{tabular}

Fig. 4: 2D Discrete Wavelet Transforms after one application 
So, the two-dimensional wavelet transforms decomposes an image into four frequency bands called subbands, each one quarter the size of the original as shown in Figure 2. LL subband contains the original image filtered and sub sampled by a factor of 2 ; the HL, $\mathrm{LH}$ and $\mathrm{HH}$ subbands contain details in the horizontal, vertical and diagonal orientations. LL subband is a coarse scale approximation of original image and the rest of frequency bands are detail signals. The transform can be applied recursively to the LL sub image to obtain decomposition at coarser scales, yielding a hierarchical decomposition or pyramid representation. The number of levels to apply depends of the original image size. The last LL band is a coarse approximation to the original image corresponding to low frequency, and all the others bands, the most part in the decomposition, are detail information corresponding to high frequency. The high frequency bands have small magnitudes and become zero if the quantization step of a loss coder is applied. So, the high frequency bands are very easy to compress.

Features extracted using DWT: The extracted features obtained by the DWT on the studied images are statistical ones and based upon the wavelet coefficients. In this section we are selected four cytological Image from Data Base (2 malign and 2 benign), for the experimental studied.

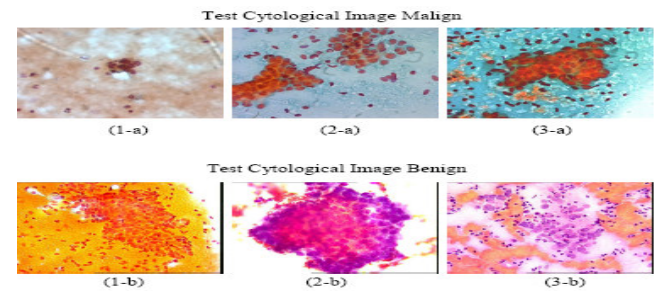

Fig. 5: Cytological Image of breast cancer for Test

Mean: It consists in the computing of the mean values of the approximated subband and the detail subbands.

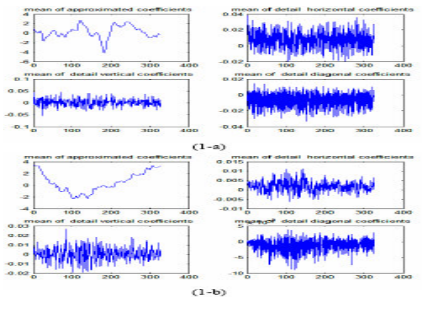

Fig. 6: Mean of the four obtained subbands using $\mathrm{db} 2$ transform for (1-a) a malign case and (1-b) a benign case
The obtained results show for begnin cases the maximum values of the mean of the approximated coefficients is bigger than the one for malign cases. Besides, for benign distribution of the mean of these coefficients there is some regularity in the obtained shapes; which is not the case of the malign samples.

Second central moment: The second central moment is the variance computed using a divisor of $n$ rather than $\mathrm{n}-1$, where $\mathrm{n}$ is the number of rows in the matrix $\mathrm{X}$. In this case, the variance is computed for all the obtained subband coefficients using Daubechies wavelet transform at level 2 and biorthogonal wavelet transform.

$m_{n}=E(x-\mu)^{2}$

Where $\mathrm{m}$ is the central moment of order 2 of the matrix $x$, and $\mu$ is the mean value.

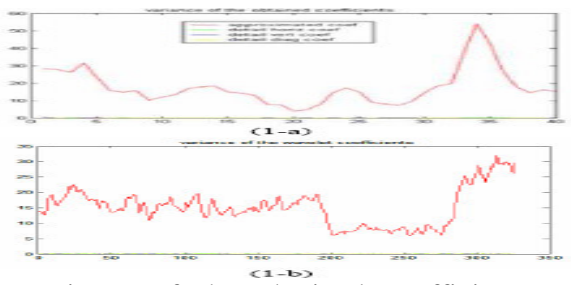

Fig. 7: Variance of the obtained coefficients using $\mathrm{db} 2$ transform of (1-a) a malign sample and (1-b) a benign sample.

The computing of the variance of the approximated coefficients for malign images using $\mathrm{db} 2$ transform and biorthogonal transform had shown a tendency of these values to have a distribution very similar to Gaussian distribution, which is not the case of benign cases.

Entropy: Entropy is a common concept in many fields, mainly in signal processing. There are different entropy criteria, many others are available and can be easily integrated. In the following expressions, $s$ is the signal and $\left(\mathrm{s}_{\mathrm{i}}\right)_{\mathrm{i}}$. Its coefficients are an orthonormal basis. The entropy $\mathrm{E}$ must be an additive cost function such that $\mathrm{E}(0)=0$ and $E(s)=\sum_{i} E\left(s_{i}\right)$.

- The normalised Shannon entropy is given by :

$$
\begin{aligned}
E 1\left(s_{i}\right) & =s_{i}^{2} \log \left(s_{i}^{2}\right) \\
E 1(s) & =-\sum_{i} s_{i}^{2} \log \left(s_{i}^{2}\right) \\
& \text { With the convention } 0 \log 0=0
\end{aligned}
$$

- The log energy entropy is given by :

$$
\begin{aligned}
& E 2\left(s_{i}\right)=\log \left(s_{i}^{2}\right) \\
& E 2(s)=\sum_{i} \log \left(s_{i}^{2}\right)
\end{aligned}
$$


Energy: This section consists in the computing of the energy percentage corresponding to the approximations vectors and the details vectors. In the following table are shown the computed values of entropy and energy obtained using a biorthogonal transform on the image for a malign sample and a benign one.

Table1: Entropy and Energy for a malign and a benign case for $\mathrm{db} 2$ transform

\begin{tabular}{lll}
\hline & Malign sample & Benign sample \\
\hline Entropy shannon & $-1.3910^{6}$ & $-6.03110^{5}$ \\
Entropy log energy & $-1.7210^{6}$ & $-4.9610^{5}$ \\
Energy app & 99.341 & 99.923 \\
Energy h & 0.05228 & 0.0076 \\
& 0.2629 & 2.0351 \\
Energy v & 0.0527 & 0.0060 \\
& 0.2568 & 1.0232 \\
Energy d & 0.0045 & 0.0006 \\
& 0.0360 & 0.0040 \\
\hline
\end{tabular}

From the obtained results, we deduce that the entropy of Shannon, the log energy entropy and the energy of approximated coefficients are, greater for the benign cases.

Discrete Wavelet packet decomposition of cytological images (DWPD method): The wavelet packet decomposition is a method based on algorithms tests the replicability in computer graphics applications. It's a generalization of the method of multi-resolution decomposition and comprises the entire family of the subband-codded tree. The decomposition leads finally to a complete wavelet tree. The original discrete texture image $I[\mathrm{~m}, \mathrm{n}]$ characterize only the original space $\mathrm{V}_{\mathrm{o}}$, while the set of approximation spaces $\left\{\mathrm{V}_{\mathrm{j}}\right\}$ is $\mathrm{V}_{\mathrm{j}-1}=\mathrm{V}_{\mathrm{j}} \otimes \mathrm{W}_{\mathrm{j}}$, where $\mathrm{W}_{\mathrm{j}}$ is the detail space. In the case of wavelet packet decomposition each projection space is denoted by the relation $\mathrm{W}_{\mathrm{j}}^{\mathrm{p}, \mathrm{q}}$, where $\mathrm{j}$ is the resolution and $\mathrm{p}, \mathrm{q}$ are subband indices, each space is a sum of four subspaces:

$$
W_{j}^{p, q}=W_{j+1}^{2 p, 2 q} \otimes W_{j+1}^{2 p+1,2 q} \otimes W_{j+1}^{2 p, 2 q+1} \otimes W_{j+1}^{2 p+1,2 q+1}
$$

The figure 8 shows the subspaces as the outputs of band-pass filters used to extract detail coefficients and detect image edge orientations along different orientations. With $A_{i}$ is an approximation subband while $D_{i}$ is corresponding to detail subband.

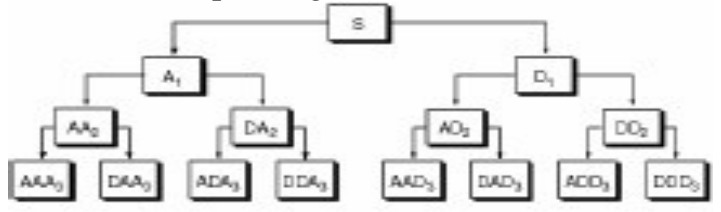

Fig. 8: Separable 8-subband filter bank
Feature extraction using DWPD of the cytological images: The objective of the feature extraction from this decomposition is to compute the vector:

$$
f=\left[\begin{array}{llllllll}
d_{x} & d_{y} & r_{x m} & r_{y m} & r_{x, s} & r_{y s} & r_{x f} & r_{y f}
\end{array}\right]
$$

Where $d_{x}$ and $d_{y}$ are measures of horizontal and

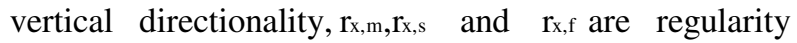

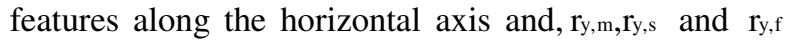
are regularity features along the vertical axis. Directionality: Directionality function along the $\mathrm{x}$ denoted by $d_{x}$ is based on the correlation between the wavelet coefficients of the successive columns in the horizontal subbands. Similarly the directionality functions along the y axis $d_{y}$ is based on the correlation between wavelet coefficients in the successive rows in the vertical subbands. To obtain the $d_{x}$ and $d_{y}$ parameters we compute in a first step the normalized lag0 -correlations $c(\mathrm{i}, \mathrm{j})$ between the rows (respectively the columns) of detail coefficients:

$$
c(i, j)=\frac{E\left(d_{i} d_{j}\right)-E\left(d_{i}\right) E\left(d_{j}\right)}{\sigma\left(d_{i}\right) \sigma\left(d_{j}\right)}
$$

Where $d_{i}$ and $d_{j}$ are sequences of the wavelet coefficients within the row $\mathrm{i}$ (or the column) and $\mathrm{j}$, $\mathrm{E}($.$) is the expected value and \sigma($.$) is the standard$ deviation. The texture is a phenomenon that depends on the region, so the edge variation estimates must be considered in a region of size $\mathrm{k}$ in the image. So that, the function $c_{k}(i)$ measuring the correlation between the rows (columns) of detail coefficients within a region of size $\mathrm{k}$ is computed:

$c_{k}(i)=\frac{1}{k} \sum_{j=i+1}^{i+k} c(i, j)$

For this experiment $\mathrm{k}=2^{1}$ where $\mathrm{l}=1,2,3,4,5$ has been applied on 3 horizontal subbands (packet $(2,1)$ or 6 , packet $(2,4)$ or 9 , packet $(2,5)$ or 10$)$ and 3 vertical ones (packet $(2,2)$ or 7 , packet $(2,8)$ or 13 , packet $(2,10)$ or 15). In figure 6 , is shown the obtained tree of a malign image using db2 wavelet packet decomposition with the Shannon entropy criterion for the optimization of the tree.

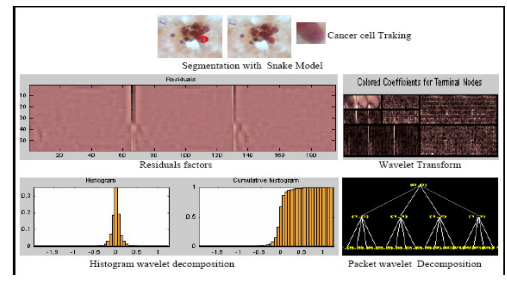

Fig.9: Packet Wavelet Decomposition of a malign sample (1-a) using db2 wavelet transform. 
The directionality $\mathrm{d}$ is obtained by integrating each sequence of $c_{k}(i)$ along the three regions of sizes 2, 4, and 8 pixels.

$d=\sum_{1, \ldots, 3} \sum_{i} c_{k}(i)$

The estimates vertical edge correlation and horizontal edge correlation for a benign sample and a malign one has been done, in the figure 10, an example of the obtained results.

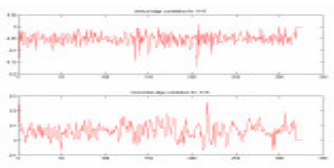

$$
\text { do (1-a) }
$$

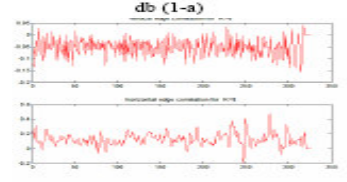

$d b(1-b)$

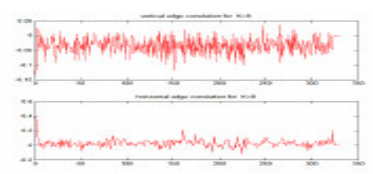

bi(1-a)

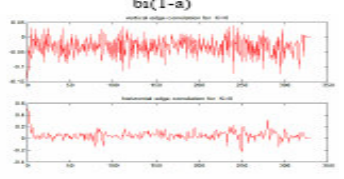

bi(1-b)
Fig. 10: - malign case $d b(1-a)$ vertical and horizontal edge correlation for a region of size 8 using db2; bi(1-a) vertical and horizontal edge correlation for a region of size 8 using bior transform.

- benign case $\mathrm{db}(1-\mathrm{b})$ vertical and horizontal edge correlation for a region of size 8 using db2; bi(1-b) vertical and horizontal edge correlation for a region of size 8 using bior transform.

The obtained results for the malign sample using both the wavelet transforms are similar. The values of vertical edge directionality are very low in the case of malignity and benignity. For horizontal edge directionality the directionality varies between 0.5 and 0.2 . This is the fact that the studied image has a non directional texture or a very limited one. In benign samples, the obtained results for vertical edge correlation and horizontal edge correlation are higher than the ones obtained for malign samples.

Regularity: Regular textures have a structural element that is repetitive in the image. The correlation $c_{k}$ has a dominant spectral component corresponding to the frequency of the structural element's repetition, so it can be used to detect the regularity of a texture. The first step is finding the maximum component of the power spectrum given by $c_{k}:\left|s_{k}\right|^{2}$ and the computing of the ratio, between the energy contained in the maximum component and the overall spectrum of energy; with $\mathrm{N}$ is the resolution used for power spectrum estimation. $\mathrm{K}=2^{1}, 1=1,2,3,4,5$. $e_{k}=\frac{\max _{N}\left|s c_{k}\right|^{2}}{\sum\left|s c_{k}\right|^{2}}$

To estimate regularity the following parameters are computed for the same previous horizontal and vertical subbands which lead to six parameters.

$r_{m}=\max _{k} e_{k}$

$r_{s}=\arg \max _{k} e_{k}$

$r_{f}=\frac{2}{N}\left(\arg \max \left|s c_{k}\right|^{2}-1\right)$

Where $r_{m}$ is the maximum value of $e_{k}, r_{s}$ is the region for which the maximum value is obtained and $\mathrm{rf}_{\mathrm{f}}$ is the normalized frequency at which $\mathrm{r}_{\mathrm{m}}$ is obtained.

Table2: Regularity parameters for a benign and a malign case

\begin{tabular}{ccccc}
\hline & \multicolumn{2}{c}{ benign } & \multicolumn{2}{c}{ malign } \\
& bior & $\mathrm{db} 2$ & bior & $\mathrm{db} 2$ \\
\hline $\mathrm{r}_{\mathrm{m}}$ & 0.6829 & 0.927 & 0.3998 & 0.8947 \\
$\mathrm{r}_{\mathrm{f}}$ & 0.0024 & -0.006 & 0.006 & -0.011 \\
$\mathrm{r}_{\mathrm{s}}$ & 0.6829 & 0.927 & 0.3998 & 0.8947 \\
\hline
\end{tabular}

Finally, the obtained regularity parameter $r_{m}$ is more important in the case of benign samples than in the malign ones. At the end of this work, eighteen textural features have been computed using methods based on Daubechies wavelet transform at level 2 and biorthogonal wavelet transform. The step of feature extraction is the first one; the next step of this project would be the classification of cytological images based on evolutionary methods such as neural networks (Multi-Layer Perceptron (MLP)).

MLP Neural Network Classification: MLP are multilayered neural network having either threshold or sigmoid activation function. The used networks in this section are three layered one, with sigmoidal activation function; the weights updating is done using mean square error minimization. The input vector to the network is a feature of parameters obtained in the later section, and the output layer has one neuron whish takes is the tumor is a benign one and 1 if it's a malign one. The used database is composed of 200 cytological images. The obtained results, has show a rate of $98 \%$ of good classification process.

\section{DISCUSSION}

In this work, the segmentation of cytological breast cancer images has been done using an active contour models process to find the tumor cell; when 
this step is achieved and cell is isolated a wavelet feature extraction approaches applied to the cell led to a vector of textural parameters. This vector is then introduced as the input vector to the MLP neural network, which is classifier in this work. Good rate of classification of the images in the database has been obtained at the end of this process.

\section{REFERENCES}

1. S. Mabrouk, J. Malek and R. Tourki, 2005: Cytological image wavelet-texture-based feature extraction: $3^{\text {rd }}$ International Conference on Systems, Signals \& Devises, pp: 341-346.

2. J. Malek, M. Abid, K. Torki and R. Tourki, 2001. Design of an efficient fuzzy clustering algorithm for breast cancer diagnosis: Smart Systems and Devices, (ISBN: 9973-31-926-5), pp: 413-418. .

3. A. Sebri, Juin 2005. Conception of system for Treatment of Cytological Image of Breast Cancer: Master's Project Specialty Microelectronics. Faculty of sciences Monastir, Tunisia, pp: 56-102.

4. W.H. Wolberg, W.N. Street and O.L. Mangasarian, 1994. Machine learning techniques to diagnose breast cancer from fine needle aspirates: Cancer Letters, vol.77, pp: 163-171.

5. W. N. Street, 2000. Xcyt: A system for remote cytological diagnosis and prognosis of breast cancer: In L.C. Jain, editor, Artificial Intelligence Techniques in Breast Cancer Diagnosis and Prognosis, World Scientific Publishing. Singapore, pp: 297-322.

6. K. Lee and W. N. Street, October 1999. A fast and robust approach for automated segmentation of breast cancer nuclei: In Proceedings of the Second IASTED International Conference on Computer Graphics and Imaging, Palm Springs, CA, pp: 4247.

7. A. Zizzari, U. Seiffert, B. Michaelis, G. Gademann and S. Swiderski, July 2001. Detection of tumor in digital images of the brain: Proceedings of the IASTED International conference, Signal processing, pp: 345-356.

8. J.C. Russ, 1999. The image processing handbook: CRC Press, Florida, $3^{\text {rd }}$ edition.

9. M.H. Bharati, J.J. Liu and J.F. Macgregor, 2004. Image texture analysis: methods and comparisons: Elsevier, Chemo metrics and Intelligent laboratory systems vol. (72): 57-71.

10. M. Kass, A. Witkin, D. Terzopoulos, 1988. Snakes: Active contour models: International Journal of Computer Vision, 1(4): 321-331.
11. Xu and J.L. Prince; December 1998. Generalized Gradient Vector Flow External Forces for Active Contours: Signal Processing, An International Journal, 71(2): 131-139.

12. $\mathrm{Xu}$ and J.L. Prince, March 2000. Global Optimality of Gradient Vector Flow: Proc. of $34^{\text {th }}$ Annual Conference on Information Sciences and Systems (CISS'00), Princeton University, pp: 122127.

13. A. Black and A. Yuille, 1993. Active Vision: Editors. IT Press.

14. T. McInerney and D. Terzopoulos, June 1995. Topologically adaptable snakes: In Proc. Of the Fifth Int. Conf. OnComputer Vision (ICCV'95), Cambridge, MA, USA, pp: 840-845.

15. R. Durikovic, K. Kaneda and H. Yamashita, 1995. Dynamic contour: a texture approach and contour operations: The Visual Computer, (11): 277-289.

16. S. R. Gunn and M. S. Nixon, January 1997. A robust snake implementation; a dual active contour: IEEE Trans. Pattern Anal. Mach. Intell, 19(1):63-68.

17. Gang Xu, E. Segawa and S. Tsuji, January 1994. Robust active contours with insensitive parameters: Pattern Recognition, 27(7):879-884.

18. R. C. Jain A. A. Amini and T. E. Weymouth, 1990. Using dynamic programming for solving variational problems in vision: IEEE Trans. on Pattern Analysis and Machine Intel. 12(9):855867.

19. A.J. Bulpitt and N. D. Efford, 1996. An efficient $3 \mathrm{~d}$ deformable model with self-optimizing mesh: Image and Vision Computing, (14):573-580.

20. L.D. Cohen, March 1991. On active contour models and balloons: CVGIP Image Understanding, (2):211-218.

21. L.V. Gool, T. Moons, E. Powrls, and A. Oosterlinck, 1995. Vision and lie's approach to invariance: Image and Vision Computing, 13(4):259-277.

22. M. Sharma, M. Markou, S. Singh, 1980. Evaluation of texture methods for image analysis: Pattern Recognition Letters, pp: 12-15.

23. S-O. Shim, T-S. Choi, 2003. Image indexing by modified color co- occurrence matrix: ICIP, IEEE.

24. M. Misiti, G. Oppenheim and J-M. Poggi, 1996. Wavelet toolbox for use with Matlab, the Math Works: Natick, MA.

25. L. Balmelli and A. Mojsilovic, October 1999. Wavelet domain features for texture description, classification and replicability analysis: Proceedings of IEEE International Conference on Image Processing ICIP, vol.(4), pp:440-444. 